\title{
Prevalence of hepatitis A viral RNA and antibodies among Chinese blood donors
}

\author{
P. Sun ${ }^{1 *}$, N. Su ${ }^{1 *}$, F.Z. Lin ${ }^{1}$, L. Ma1 ${ }^{1}$, H.J. Wang ${ }^{2}$, X. Rong ${ }^{3}$, Y.D. Dai ${ }^{4}, \mathrm{~J} \mathrm{Li}^{5}$, \\ Z.W. Jian ${ }^{6}$, L.H. Tang ${ }^{7}$, W. Xiao' ${ }^{1}$ and C.Q. Li ${ }^{1}$ \\ ${ }^{1}$ Institute of Blood Transfusion, Chinese Academy of Medical Sciences, \\ Chengdu, China \\ ${ }^{2}$ Blood Center of the Beijing Red Cross, Beijing, China \\ ${ }^{3}$ Guangzhou Blood Center, Guangzhou, China \\ ${ }^{4}$ Blood Center of the Nanjing Red Cross, Nanjing, China \\ ${ }^{5}$ Shaanxi Blood Center, Xian, China \\ ${ }^{6}$ Deyang Center Blood Station, Deyang, China \\ ${ }^{7}$ Suzhou Center Blood Station, Suzhou, China \\ ${ }^{*}$ These authors contributed equally to this study. \\ Corresponding authors: F.Z. Lin / C.Q. Li \\ E-mail: Ifangzhao@126.com / lichangqing268@163.com
}

Genet. Mol. Res. 14 (4): 16431-16437 (2015)

Received August 10, 2015

Accepted October 2, 2015

Published December 9, 2015

DOI http://dx.doi.org/10.4238/2015.December.9.13

\begin{abstract}
Like other developing countries, China was reported to have a relatively high seroprevalence of anti-hepatitis $A$ antibodies (anti-HAV). However, no studies have evaluated the prevalence of anti-HAV and HAV RNA among voluntary blood donors with or without elevated serum alanine transaminase (ALT) levels. Anti-HAV antibodies were detected using an enzyme-linked immunosorbent assay, and reverse transcription quantitative polymerase chain reaction was carried out for detection of HAV RNA. In the current study, we analyzed a total of 450 serum samples with elevated ALT levels ( $\geq 40 \mathrm{U} / \mathrm{L}$ ) and 278 serum samples with non-elevated ALT levels. Seroprevalence rates of anti-HAV were $51.6 \%$ in donors with elevated ALT and $41.4 \%$ in donors with non-elevated ALT; however, none of the samples was positive for HAV RNA. The results of our study showed lower seroprevalence rates of anti-HAV in blood donors (irrespective of
\end{abstract}


ALT levels) than those in published data on Chinese populations. Although donors with elevated ALT had statistically higher prevalence rates of antiHAV than did those with non-elevated ALT, none of the serum samples had detectable levels of the active virus. In conclusion, our results demonstrate that the transmission of hepatitis A by blood transfusion will occur rarely.

Key words: Hepatitis A Virus; HAV; anti-HAV antibodies; HAV-RNA; HAV prevalence

\section{INTRODUCTION}

Hepatitis A virus (HAV) infection is the most common form of acute viral hepatitis worldwide. It is estimated that 1.5 million of HAV infections occur each year. HAV is highly contagious and usually spread by the fecal-oral route (Wasley et al., 2006). However, recent studies have shown that HAV can be transmitted by blood transfusion when viruses are present in the blood of donors (Brundage and Fitzpatrick, 2006). Like other developing countries, China was reported to have a relatively high seroprevalence of anti-HAV antibodies (Geng et al., 1998). Despite this information, HAV vaccination has only been listed as one of the Expanded Program on Immunization vaccines since 2008.

Alanine aminotransferase (ALT) is abundant in liver cells, and elevated serum ALT levels in blood donors have been associated with an increased risk of post-transfusion hepatitis (PTH). Thus many countries, including China, have compulsory ALT testing when screening blood donors (Alter et al., 1981; Chang et al., 2007; Kim et al., 2008; MOH, 2012). As such, a great number of donors are deferred because of elevated ALT levels (Kim et al., 2008). However, the efficiency of ALT testing to reduce hepatitis transmission is controversial because there are many other causes of elevated serum ALT levels (Bizzaro et al., 1992; Ramesh et al., 1995). Serological testing for the detection of antibodies to certain viruses is used to confirm infection. Chinese blood centers screen donors by testing for hepatitis B surface antigen (HBsAg) and antibodies to hepatitis $\mathrm{C}$ virus (anti-HCV) to reduce the risk of PTH (Ren et al., 2011). During the past two to three decades, a more sensitive screening method known as nucleic acid amplification techniques (NAT) has been introduced in many countries to detect infectious donors during the serological window period (Li et al., 2008). However, this method has not yet been implemented in China. Currently, there are many pilot studies evaluating NAT for the detection of human immunodeficiency virus (HIV), HBV, and hepatitis $\mathrm{C}$ virus (HCV) in Chinese blood donors (Shan et al., 2007).

In the current study, we investigated the seroprevalence of hepatitis A virus antibodies among Chinese blood donors with or without elevated ALT levels, and for the first time, we screened the donors with NAT for HAV. Our objective was to determine whether a relationship exists between ALT and HAV infection and to evaluate the possibility of blood-borne transmission of HAV in China.

\section{Material and methods}

\section{Source population}

Serum samples were collected from the following blood centers between March 2013 and July 2013: Suzhou Central Blood Station, Beijing Blood Center, Nanjing Blood Center, Shanxi Blood Center, Guangzhou Blood Center, and Deyang Central Blood Station. In this study, we analyzed 450 serum samples with elevated ALT levels ( $\geq 40 \mathrm{U} / \mathrm{L}$ as measured via the rate method) and 
278 serum samples with no elevated ALT levels ( $<40 \mathrm{U} / \mathrm{L}$ as measured via the rate method).

All serum samples were negative for HBsAg, anti-HCV, Treponema pallidum, and HIV. All studies on human subjects were performed in accordance with the ethical standards of the committee on Human Experimentation of Red Cross Society of China.

\section{Detection of antibodies to HAV}

To detect anti-HAV, enzyme-linked immunosorbent assays (ELISA) were carried out using anti-HAV ELISA kits (Beifang Institute of Biotechnology, Beijing, China) according to manufacturer instructions. The ELISA plates were read on an automated microplate reader (Molecular Devices Corporation, USA).

\section{NAT for HAV RNA}

Reverse transcription quantitative PCR (RT-qPCR) was carried out for detection of HAV RNA in all serum samples. Nucleic acid isolation was performed using a viral RNA extraction kit (Daan Gene Co., Guangzhou, China) according to manufacturer instructions. Then PCR amplification of HAV gene was performed with forward primer (5'-TTTCTTCCAGGGCTCTCC-3'), reverse primer (5'-CTAATCATGGAGTTGACCCC-3'), and probe (5'-FAM-TTGCCCTAGGCTCTGGCCGT T-3'-TAMRA). ABI StepOnePlus Detection System (Applied Biosystems, USA) was used for amplification, data acquisition, and analysis.

\section{Statistical analysis}

All statistical analyses were performed using the SPSS statistical analysis software package. Differences were considered significant if $P<0.05$.

\section{Results}

\section{Study participation}

During the study period, 728 blood donors were enrolled. A total of $81 \%$ (590/728) of study participants were male. Ages of the donors ranged from 18 to 57 years and the mean age was 24.5 years. The differences between the two groups (with or without elevated ALT levels) were not statistically significant $\left(X^{2}=0.076, P>0.05\right)$

\section{Antibodies to HAV testing}

Testing for anti-HAV was performed on all the samples. In the elevated ALT group, 232 samples were found to be positive for anti-HAV, corresponding to a prevalence of $51.6 \%$. Among the 278 serum samples without elevated ALT levels, 115 (41.4\%) were found to be positive for anti-HAV (Table 1). As seen in Figure 1 and Figure 2, the prevalence of anti-HAV generally increased with age irrespective of ALT levels, and the atypical decline ( $\leq 40$ group with elevated ALT levels and $\leq 60$ without elevated ALT levels) may be attributed to the sample numbers. Then we compared the prevalence of anti-HAV between men and women of different age groups (irrespective of ALT levels). As seen in Figure 3, women had lower prevalence of anti-HAV than did men. 
Table 1. Prevalence of anti-HAV in the blood donors.

\begin{tabular}{lccr}
\hline & ALT $\geq 40$ U/L & ALT < 40 U/L & Published studies + \\
\hline Prevalence (\%) & $232 / 450(51.6 \%)$ & $115 / 278(41.4 \%)$ & $1 / 124(0.8 \%)$ \\
\hline
\end{tabular}

+All comparison values were based on blood donor population samples (Notari EP et al., 2001).

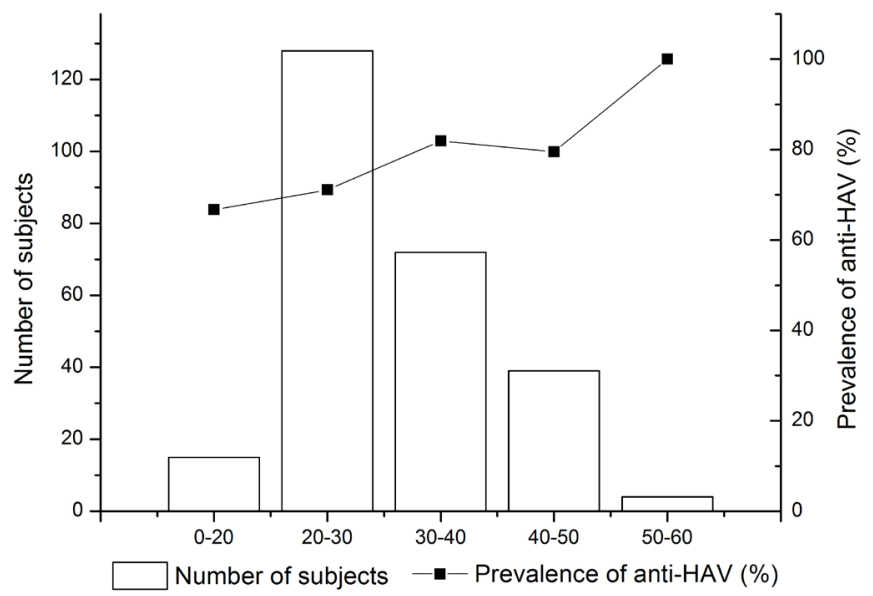

Figure 1. Prevalence of anti-HAV in Chinese blood donors based on ALT levels and characterization of anti-HAV with elevated ALT levels by age range. Bars represent the number of subjects in each group, indicated by the left vertical axis, and the points represent the prevalence in each group, indicated by the right vertical axis. The age ranges are delineated on the horizontal axis.

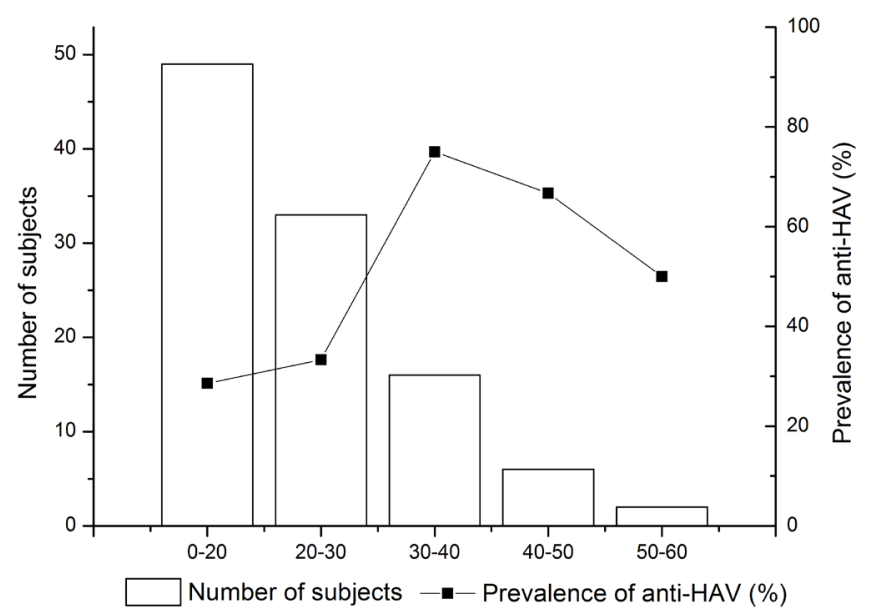

Figure 2. Prevalence of anti-HAV in Chinese blood donors based on ALT levels and characterization of anti-HAV without elevated ALT levels by age range. Bars represent the number of subjects in each group, indicated by the left vertical axis, and the points represent the prevalence in each group, indicated by the right vertical axis. The age ranges are delineated on the horizontal axis. 


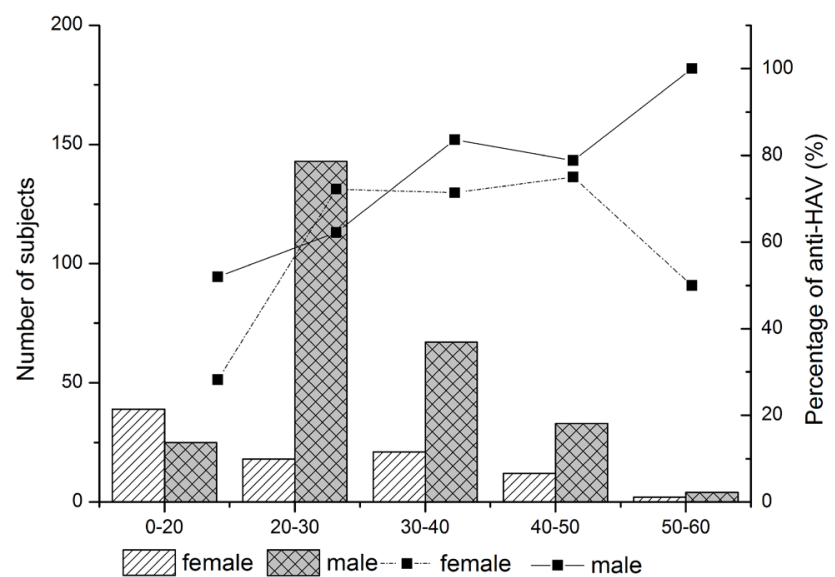

Figure 3. Prevalence of anti-HAV in Chinese blood donors based on gender. Bars represent the number of subjects in each group, indicated by the left vertical axis, and the points represent the prevalence in each group, indicated by the right vertical axis. The age ranges are delineated on the horizontal axis.

\section{NAT for HAV RNA}

HAV RNA testing was completed on all the 728 study samples. None of the 728 samples was tested positive for HAV RNA.

\section{DISCUSSION}

Hepatitis A virus is one of the most common causative agents for acute hepatitis worldwide, which accounts for approximately one quarter of clinical hepatitis cases (Yotsuyanagi et al., 1996; Kwon et al., 2000; Hussain et al., 2011). Seroprevalence of anti-HAV is highly correlated with socioeconomic status and access to clean water and sanitation (Jacobsen and Koopman, 2004). Moreover, high anti-HAV seroprevalence rates have been reported in developing countries (Geng et al., 1998; Sawayama et al., 1999; Arankalle et al., 2001). The results of the current study showed lower seroprevalence rates $(347 / 728,47.7 \%)$ of anti-HAV in blood donors (irrespective of ALT levels) than those in published data regarding Chinese populations (Geng et al., 1998). This decline may be attributed to two reasons: improvements in socioeconomic status and the consistency of voluntary blood donors (the majority of whom are males between the age of 18 and 35 years old). However, the seroprevalence rates found in our study are still much higher than those in published data about blood donors in the United States (Notari et al., 2001). The differences may also be due to sample sizes, study populations, testing methods, and geography. Once infected by $\mathrm{HAV}$, lifelong immunity develops; hence, seroprevalence rates of anti-HAV increase with age, as was found in our study. The data in the present study also indicate that the prevalence rate of anti-HAV is higher in males than in females $(69 \%$ males versus $53 \%$ females, $P<0.05)$. This could be attributed to a more frequent exposure to sources of contamination, perhaps owing to greater activity in the labor market, involvement in high-risk work in raising animals, or cleaning services in the sewerage disposal system.

The transmission of HAV by blood products has been thought to occur rarely or not at all. However, several cases of HAV transmission by transfusion of blood products have been 
reported in the last three decades (Noble et al., 1984; Sherertz et al., 1984; Lemon, 1994; Mosley et al., 1994). The explanation for the small number of reported cases of HAV transmitted by transfusion is the lack of an asymptomatic carrier state for hepatitis $A$, in contrast to hepatitis $B$. Thus, unlike HBV carriers who may remain infectious for years, the infectious period for a patient with HAV would be limited to the 1- or 2-week viremic period prior to the onset of symptoms or jaundice. Studies have demonstrated that viremia of HAV lasts approximately 6 weeks (Bower et al., 2000). This may explain why no HAV RNA was detected in this study. Our results also showed that all serum samples no longer had detectable levels of active virus. In this respect, although the donors with elevated ALT had a statistically higher prevalence rate of anti-HAV than did those with non-elevated ALT $\left(X^{2}=9.968, P=0.02\right)$, all serum samples showed little possibility to transmit HAV.

China belongs to a group of hepatitis high-risk countries and great considerations should be given to prevent transfusion-transmitted hepatitis. Our results assure that concentration should be placed on other hepatitis viruses, such as $\mathrm{HBV}, \mathrm{HCV}$, and new hepatitis viruses. Conventional ALT testing causes a large number of normal blood donors to be excluded. This can cause not only serious economic losses, but also undue psychological stress to those excluded as blood donors. Further studies are needed to determine whether ALT testing should be cancelled and more direct and sensitive methods to detect hepatitis should be adopted.

\section{Conflicts of interest}

The authors declare no conflicts of interest.

\section{ACKNOWLEDGMENTS}

We thank Haijun Cao, Zhongkui Wang, Shengliang Ye, and Fengjuan Liu for excellent technical assistance. The secretarial assistance of Rong Zhang, Bing Liu, Ye Xin, and Xianjiao Zheng is also greatly appreciated.

\section{REFERENCES}

Alter HJ, Purcell RH, Holland PV, Alling DW, et al. (1981). Donor transaminase and recipient hepatitis. Impact on blood transfusion services. JAMA 246: 630-634.

Arankalle VA, Chadha MS, Chitambar SD, Walimbe AM, et al. (2001). Changing epidemiology of hepatitis A and hepatitis E in urban and rural India (1982-98). J. Viral. Hepat. 8: 293-303.

Bizzaro N, Tremolada F, Casarin C, Bonetti P, et al. (1992). Serum alanine aminotransferase levels among volunteer blood donors: effect of sex, alcohol intake and obesity. Ital. J. Gastroenterol. 24: 237-241.

Bower WA, Nainan OV, Han X and Margolis HS (2000). Duration of viremia in hepatitis A virus infection. J. Infect. Dis. $182: 12-17$. Brundage SC and Fitzpatrick AN (2006). Hepatitis A. Am. Fam. Physician 73: 2162-2168.

Chang Y, Ryu S, Sung E and Jang Y (2007). Higher concentrations of alanine aminotransferase within the reference interval predict nonalcoholic fatty liver disease. Clin. Chem. 53: 686-692.

Geng J, Xu D, Gong J and Li W (1998). Assessing hepatitis A virus epidemic stochastic process in eight cities in China in 1990. Int. J. Epidemiol. 27: 320-322.

Hussain Z, Husain SA, Almajhdi FN and Kar P (2011). Immunological and molecular epidemiological characteristics of acute and fulminant viral hepatitis A. Virol. J. 8: 254.

Jacobsen KH and Koopman JS (2004). Declining hepatitis A seroprevalence: a global review and analysis. Epidemiol. Infect. 132: 1005-1022.

Kim WR, Flamm SL, Di Bisceglie AM and Bodenheimer HC (2008). Serum activity of alanine aminotransferase (ALT) as an indicator of health and disease. Hepatology 47: 1363-1370. 
Kwon OS, Byun KS, Yeon JE, Park SH, et al. (2000). Detection of hepatitis A viral RNA in sera of patients with acute hepatitis A. J. Gastroenterol. Hepatol. 15: 1043-1047.

Lemon SM (1994). The natural history of hepatitis A: the potential for transmission by transfusion of blood or blood products. Vox Sang 67 19-23; discussion 24-16.

Li L, Chen PJ, Chen MH, Chak KF, et al. (2008). A pilot study for screening blood donors in Taiwan by nucleic acid amplification technology: detecting occult hepatitis $\mathrm{B}$ virus infections and closing the serologic window period for hepatitis $\mathrm{C}$ virus. Transfusion 48: 1198-1206.

Ministry of Health of the People's Republic of China CNSM (2012). Quality requirements for whole blood and blood components. $1-13$.

Mosley JW, Nowicki MJ, Kasper CK, Donegan E, et al. (1994). Hepatitis A virus transmission by blood products in the United States. Transfusion Safety Study Group. Vox Sang 67: 24-28.

Noble RC, Kane MA, Reeves SA and Roeckel I (1984). Posttransfusion hepatitis A in a neonatal intensive care unit. JAMA 252: 2711-2715.

Notari EP, Orton SL, Cable RG, Grindon AJ, et al. (2001). Seroprevalence of known and putative hepatitis markers in United States blood donors with ALT levels at least 120 IU per L. Transfusion 41: 751-755.

Ramesh V, Saraswat S, Choudhury N and Gupta RK (1995). Relationship of serum alanine aminotransferase (ALT) to body mass index (BMI) in blood donors: the need to correct ALT for BMI in blood donor screening. Transfus. Med. 5: $273-274$.

Ren FR, Wang JX, Huang Y, Yao FZ, et al. (2011). Hepatitis B virus nucleic acid testing in Chinese blood donors with normal and elevated alanine aminotransferase. Transfusion 51: 2588-2595.

Sawayama Y, Hayashi J, Ariyama I, Furusyo N, et al. (1999). A ten year serological survey of hepatitis A, B and C viruses infections in Nepal. J. Epidemiol. 9: 350-354.

Shan H, Ren FR, Zhao HY, Zhang YZ, et al. (2007). A multi-Chinese blood center study testing serologic-negative donor samples for hepatitis $C$ virus and human immunodeficiency virus with nucleic acid testing. Transfusion 47: $2011-2016$.

Sherertz RJ, Russell BA and Reuman PD (1984). Transmission of hepatitis A by transfusion of blood products. Arch. Intern. Med. 144: 1579-1580.

Wasley A, Fiore A and Bell BP (2006). Hepatitis A in the era of vaccination. Epidemiol. Rev. 28: 101-111.

Yotsuyanagi H, Koike K, Yasuda K, Moriya K, et al. (1996). Prolonged fecal excretion of hepatitis A virus in adult patients with hepatitis $\mathrm{A}$ as determined by polymerase chain reaction. Hepatology 24: 10-13. 